\title{
ENGEVISTA
}

Página da revista: http://www.uff.br/engevista/seer/

\section{Planejamento fatorial para otimização das condições da reação de transesterificação alcalina do óleo de pequi (Caryocar brasiliense Cambess)}

\author{
Ana Paula Arruda ${ }^{1}$ \\ Isabel Matos Fraga ${ }^{2}$ \\ Demétrio de Abreu Sousa ${ }^{3}$ \\ Danilo Morais Itokagi ${ }^{4}$ \\ Cleyton Souza Pereira ${ }^{5}$ \\ Neuza Gomes Pereira ${ }^{6}$
}

Resumo: O presente estudo avaliou a influência de três variáveis, catalisador (C) $\mathrm{NaOH}$ e $\mathrm{KOH}$, temperatura de 60 e $80^{\circ} \mathrm{C}$ e razão molar álcool/óleo (R) de 6:1 e 12:1, na transesterificação do óleo de pequi por meio de um planejamento fatorial $2^{3}$. Todas as variáveis foram avaliadas em seus níveis máximos e mínimos. Os resultados demonstraram que o maior rendimento em ésteres correspondeu a 73\%, utilizando catalisador (C) KOH, razão molar (R) de 12:1 (etanol:óleo) e temperatura de $60^{\circ} \mathrm{C}$, sendo que rendimentos ligeiramente menores $37,25 \%$ sem significância foram obtidos com o emprego $\mathrm{NaOH}$, razão molar 12:1 (etanol:óleo) e a $80^{\circ} \mathrm{C}$. Separadamente a variável de maior importância para a transesterificação do óleo de pequi foi a temperatura (T) em nível menor $\left(60^{\circ} \mathrm{C}\right)$ e o catalisador não apresentou efeito significativo no rendimento da reação. A partir dos cálculos de efeito da combinação entre as três variáveis, observou-se que os melhores rendimentos são encontrados quando se utiliza o catalisador em seu maior nível $(\mathrm{KOH})$, combinado com níveis contrários de razão molar e temperatura. Ou seja, os melhores rendimentos são encontrados quando a transesterificação é realizada utilizando como catalisador o $\mathrm{KOH}$, uma razão molar de $12: 1$ e uma temperatura de $60^{\circ} \mathrm{C}$, ou como catalisador o $\mathrm{KOH}$, uma razão molar de 6:1 e uma temperatura de $80^{\circ} \mathrm{C}$.

\footnotetext{
${ }^{1}$ IFMT - Instituto Federal de Educação, Ciência e Tecnologia de Mato Grosso

${ }^{2}$ IFMT - Instituto Federal de Educação, Ciência e Tecnologia de Mato Grosso

${ }^{3}$ IFMT - Instituto Federal de Educação, Ciência e Tecnologia de Mato Grosso

${ }^{4}$ IFMT - Instituto Federal de Educação, Ciência e Tecnologia de Mato Grosso

${ }^{5}$ IFMT - Instituto Federal de Educação, Ciência e Tecnologia de Mato Grosso

${ }^{6}$ IFMT - Instituto Federal de Educação, Ciência e Tecnologia de Mato Grosso
} 
Palavras-chave: Biodiesel, pequi, planejamento fatorial, transesterificação.

\begin{abstract}
This study evaluated the influence three variables, catalyst $(\mathrm{C}), \mathrm{NaOH}$ and $\mathrm{KOH}$, a temperature of 60 to $80^{\circ} \mathrm{C}$ and the molar ratio ethanol / oil (A) 6: 1 and 12: 1, Pequi oil transesterification through a factorial design 23. All variables were assessed at their maximum and minimum levels. The results showed that the highest yield corresponded to $73 \%$ ester, using catalyst $(\mathrm{C}) \mathrm{KOH}$, mole ratio (R) of 12: 1 (ethanol: oil) and $60{ }^{\circ} \mathrm{C}$, slightly lower yields $37.25 \%$ without statistical significance was obtained by employing $\mathrm{NaOH}$, molar ratio 12: 1 (ethanol: oil) and $80^{\circ} \mathrm{C}$. Separately the variable of greatest importance to the pequi oil transesterification was the temperature $(\mathrm{T})$ in lower level $\left(60^{\circ} \mathrm{C}\right)$ and the catalyst had no significant effect on the yield of the reaction. From the calculations of the effect of combination of the three variables, it was observed that the best yields are found when using the catalyst in its highest level $(\mathrm{KOH})$ combined with opposite levels molar ratio and temperature. That is, the best yields are found when the transesterification is carried out using as catalyst $\mathrm{KOH}$, a molar ratio of 12: 1 and a temperature of $60^{\circ} \mathrm{C}$, or as catalyst $\mathrm{KOH}$, a molar ratio of 6: 1 and a temperature of $80^{\circ} \mathrm{C}$.
\end{abstract}

Keywords: Biodiesel, pequi, factorial design, transesterification. 


\section{Introdução}

O biodiesel surgiu como um combustível alternativo para motores do ciclo diesel capaz de proporcionar uma redução na emissão de gases do efeito estufa, bem como dar autonomia a países dependentes de outros em relação ao petróleo (Ferrari et al. 2005).

O biodiesel é definido como um combustível renovável, já que predominantemente, é produzido a partir de óleos vegetais ou gorduras animais. A síntese desse combustível se dá pela reação desses óleos com um álcool de cadeia curta, mais comumente, metanol e etanol.

No Brasil, predomina para a produção de biodiesel, a reação denominada transesterificação. Nessa reação, um mol de óleo reage com no mínimo três mols de um álcool de cadeia curta, impulsionados por um catalisador que pode ser ácido ou básico. O uso de catalisadores básicos, geralmente hidróxido de potássio, é muito mais comum devido ao fato de proporcionar um processo de produção mais barato e fornecer maiores rendimentos do produto (Knothe et al. 2006; Geris et al. 2007).

Muitas são as matérias-primas utilizadas para produção de biodiesel. Geralmente a escolha da matéria-prima se deve a condições climáticas e geográficas do país onde se processa a produção.

No Brasil, não é de se estranhar a predominância do uso do óleo de soja, seguido do sebo bovino para a síntese de biodiesel, já que se trata de um país que se destaca na produção de soja e gado de corte.

No entanto, devido ao aumento da incorporação do biodiesel ao diesel em 2014, muitas matériasprimas têm sido investigadas, quanto ao seu potencial para produção de biodiesel, dentre elas pode-se destacar o óleo extraído do pequi (Caryocar brasiliense).

O pequi é um fruto sazonal com safra entre os meses de novembro e fevereiro, dependendo da região. Altamente rentável, para os agricultores do cerrado brasileiro, chamou a atenção dos pesquisadores que passaram a desenvolver projetos voltados para o processamento da polpa de pequi e a melhoria da qualidade de seus derivados, como o óleo de pequi, que tem sido bastante estudado (Lima et al. 2007).

O principal produto do pequizeiro é a polpa (mesocarpo interno), muito utilizada na culinária regional. O mesocarpo interno contém óleos que são utilizados como condimentos, na fabricação de licores, na indústria de lubrificantes e de cosméticos (sabão, sabonete e cremes). A polpa de pequi apresenta teores de lipídicos que variam de 20 a $27 \%$ (Lopes et al. 2006).

No entanto, a atual demanda de produção de biodiesel requer condições de reações que proporcionem um melhor aproveitamento da matéria-prima na síntese de biodiesel, consequentemente uma maior conversão de óleo em ésteres.

Muitos são os trabalhos que tem sido realizado utilizando o óleo de pequi como matéria-prima para a produção de biodiesel, principalmente na síntese de biodiesel metílico via catálise básica, os resultados encontrados têm sido satisfatórios.

Portanto, a reação de transesterificação do óleo de pequi já é conhecida, porém há a necessidade de encontrar as melhores condições de reação do referido óleo para que se obtenha uma maior conversão do óleo em ésteres. Condições como temperatura, catalisador e razão molar álcool/óleo podem ser decisivas na obtenção de uma boa conversão de óleos em biodiesel. 
Diante do exposto este trabalho tem por objetivo avaliar a melhor condição para obtenção de uma maior conversão do biodiesel produzido a partir da transesterificação alcalina do óleo extraído do caroço de pequi, através de um estudo utilizando a planejamento fatorial $2^{3}$ com três fatores variáveis (catalisador, razão molar e temperatura) cada qual apresentando dois níveis de estudo.

\section{Metodologia}

O presente trabalho foi desenvolvido no Laboratório de Química do Instituto Federal de Educação, Ciência e Tecnologia do Mato Grosso - Campus Cáceres. O óleo refinado do Pequi (Caryocar brasiliense Cambess) foi adquirido na empresa Mundo dos óleos.

\subsection{Caracterização do óleo de pequi}

O óleo de pequi foi caracterizado quanto a seu índice de acidez, densidade, umidade e porcentagem de ácidos graxos, todas essas análises foram realizadas de acordo com a metodologia descrita por Moretto e Alves, (1986) e Moura (2010). Conforme descrito a seguir.

\subsection{1. Índice de acidez}

$\mathrm{O}$ índice de acidez corresponde ao número de miligramas de $\mathrm{KOH}$ necessário para neutralizar a acidez de $1 \mathrm{~g}$ de uma amostra de óleo ou gordura (mg KOH/g), sendo essa acidez reflexo da presença de ácidos graxos livres na amostra. O procedimento consiste em colocar dois gramas da amostra em um erlenmeyer adicionando-se em seguida $25 \mathrm{~mL}$ de solução de éter etílico: etanol (2:1) para esse recipiente, agitando-se vigorosamente e adicionando-se a seguir duas gotas de solução alcoólica de fenolftaleína a 1\%, e, por fim, titulando-se com solução aquosa de hidróxido de sódio $0,1 \mathrm{~N}$ até viragem do indicador de incolor para uma tonalidade rósea. O cálculo da acidez é determinado pela equação 1. (Moretto; Alves, 1986).

$$
\mathrm{AC}=\frac{\mathrm{V} \times 5,61 \times \mathrm{F}}{\mathrm{M}}
$$

Onde:

$\mathrm{AC}=$ índice de acidez;

$\mathrm{V}=$ volume de $\mathrm{NaOH}$ gasto para titular a amostra;

5,61 = é o equivalente grama do $\mathrm{KOH}$;

$\mathrm{F}=$ fator de correção de $\mathrm{NaOH}$;

$M=$ massa da amostra.

\subsubsection{Porcentagem de ácidos graxos}

A determinação da porcentagem de ácidos graxos livres foi realizada de acordo com a metodologia descrita por Moretto e Alves (1986), que determina a porcentagem de ácidos graxos livres, expressa como ácido oleico, em óleos comuns, brutos e refinados. Foram pesados aproximadamente $5 \mathrm{~g}$ da amostra em um 
erlenmeryer e foram adicionados $50 \mathrm{~mL}$ de álcool etílico (95,8\% de pureza), previamente neutralizado com solução aquosa de $\mathrm{NaOH} 0,1 \mathrm{~N}$, utilizando $0,5 \mathrm{~mL}$ de solução etanoica de fenolftaleína a $1 \%$ como indicador. Em seguida, aqueceu-se a solução sobre uma placa térmica até apresentar "sinais" de ebulição. Depois, titulou-se ainda quente com solução aquosa de $\mathrm{NaOH} 0,1 \mathrm{~N}$, até coloração rósea persistente por 15 segundos. O cálculo da porcentagem de ácidos graxos livres foi determinado pela equação 2 .

$$
A G L=\left(\frac{V * F * 28,2}{P}\right)
$$

Onde:

$\mathrm{V}=$ número de $\mathrm{mL}$ de solução de hidróxido de sódio a $0,1 \mathrm{~N}$ gasto na solução;

$\mathrm{F}=$ fator da solução de hidróxido de sódio;

$\mathrm{P}=$ número de gramas da amostra;

\subsubsection{Umidade e materiais voláteis}

A determinação da umidade foi efetuada através do método de perdas por dessecação em estufa de acordo com o método AOCS Bc 2-49. Cerca de $5 \mathrm{~g}$ de cada amostra foram pesadas em cadinhos de porcelana e aquecidas a $130^{\circ} \mathrm{C}$ em estufa. Após o aquecimento, as amostras foram imediatamente tampadas e resfriadas em dessecador até atingirem a temperatura ambiente e, em seguida, foram novamente pesadas. O teor de umidade e matéria volátil foi determinado pela equação 3 (Moura, 2010).

$$
\mathrm{UMV} \%=\frac{(\text { perda de peso } * 100)}{(\text { peso da amostra })}
$$

Onde:

UMV = umidade e matéria Volátil

\subsubsection{Densidade}

A determinação da densidade foi realizada de acordo com a metodologia descrita por Moura, (2010). Foi utilizado um picnômetro previamente calibrado com substância de densidade conhecida. O picnômetro de 30, $42 \mathrm{~mL}$ teve seu peso vazio aferido. Posteriormente adicionou-se o óleo ao picnômetro e o mesmo cheio, foi novamente pesado, esse procedimento foi realizado em triplicata. A densidade relativa foi determinada através da equação 4.

$$
\text { Densidade }=\frac{m-m_{p}}{V}
$$

Onde:

M=é a massa do picnômetro com óleo,

$\mathrm{m}_{\mathrm{P}=}$ é a massa do picnômetro vazio e 
V=é o volume do picnômetro.

\subsection{Planejamento fatorial}

A fim de determinar o número de experimentos necessários para avaliar os efeitos do tipo de catalisador, temperatura de reação e razão molar álcool/óleo, no rendimento da reação, foi utilizado um planejamento fatorial completo $2^{3}$ com duas repetições. O planejamento fatorial é útil quando um grande número de fatores pode ser avaliado simultaneamente, calculando-se os efeitos principais e de interações entre os fatores com um reduzido número de experimentos (Sousa, 2014).

A organização de um planejamento fatorial consiste em selecionar os fatores (variáveis independentes do sistema) e escolher os níveis (valores assumidos pelas variáveis) que serão estudados. De acordo com (Cunico et al. 2007), fatores são definidos como a variável do sistema que se quer analisar. Níveis são definidos como a condição em que os fatores irão operar durante os experimentos. Nesse estudo adotou-se a simbologia de níveis máximos (+) e níveis mínimos (-). Os efeitos a serem calculados representam a influência de cada fator no rendimento do biodiesel produzido.

A determinação da quantidade de experimentos é feita de acordo com a quantidade de variáveis estudadas e com os níveis estipulados para essas variáveis. O planejamento é representado na forma de potência, fornecendo assim o número de experimentos a serem realizados (Lima, 2007).

Para determinar o efeito e a influência dos níveis de cada fator no sistema proposto, estes níveis foram codificados como +1 e -1, para o maior e menor nível, respectivamente. Os três fatores escolhidos no planejamento desse trabalho foram: (1). Catalisador (2) Razão molar álcool/óleo e (3) Temperatura. Cada fator foi utilizado em seu nível mínimo (-1) e seu nível máximo (-2), as especificações desses níveis são apresentadas na tabela 1. Foram realizados, 8 experimentos em duplicata totalizando 16 experimentos para avaliação da combinação das melhores condições para obtenção de uma maior conversão de biodiesel.

Tabela 1. Planejamento fatorial $2^{3}$ - Variáveis em seus níveis máximos e mínimos

\begin{tabular}{c|c|c}
\hline Variáveis & Nível máximo (+1) & Nível mínimo (-1) \\
\hline Catalisador & $\mathrm{KOH}$ & $\mathrm{NaOH}$ \\
Razão molar álcool/óleo & $12: 1$ & $6: 1$ \\
Temperatura & $80^{\circ} \mathrm{C}$ & $60^{\circ} \mathrm{C}$ \\
\hline
\end{tabular}

O efeito principal é calculado como uma média dos efeitos individuais permitindo a avaliação média do efeito de cada fator separadamente das condições dos efeitos dos outros fatores. O cálculo do efeito principal é feito a partir da equação 5 (Cunico et al. 2007).

$$
\text { Efeito principal }=2\left(\sum \mathrm{y}+-\sum \mathrm{y}-\right) /(\mathrm{ba})
$$

Onde,

$\mathrm{y}=$ média dos efeitos individuais em cada medida, sinal (+) e (-) = representam os níveis de cada fator $\mathrm{ba}=$ representa o número total de experimentos. 
De acordo com Sousa, (2014), os efeitos de interações levam em conta os fatores combinados uns com os outros. Neste caso, foram calculados os efeitos de interação de $2^{\mathrm{a}}$ ordem (dois fatores entre si) e $3^{\mathrm{a}}{ }_{-}$ ordem (três fatores entre si). Os efeitos de interações de $2^{\mathrm{a}}$-ordem e $3^{\mathrm{a}}$-ordem foram calculados usando as equações 6 e 7 , respectivamente.

Interações de $2^{\text {a }}$-ordem

$$
2\left[\left(\sum \mathrm{y}^{++}+\sum \mathrm{y}^{--}\right)-\left(\sum \mathrm{y}^{-+}+\sum \mathrm{y}^{+-}\right)\right] /(\mathrm{ba})
$$

Interações de $3^{\mathrm{a}}$ ordem

$$
2[(y+--+y-+-+y--++y+++)-(y---+y++-+y+-++y-++)] /(b a)
$$

Depois de calcular todos os efeitos, um gráfico de 'probabilidade normal vs efeito' foi plotado com os valores de probabilidade para uma distribuição normal versus o valor calculado de cada efeito, com o objetivo de visualizar quais os fatores possuem efeitos mais proeminentes. Posteriormente calculou-se a variância experimental (eq. 8), o erro experimental (eq. 9), a variância dos efeitos (eq. 10) e o erro dos efeitos (eq. 11). Então, aplicou-se o teste t de Student para comparar as variâncias, erros experimentais e os efeitos. Um t-crítico pode ser obtido pela multiplicação do valor tabelado de $\mathrm{t}$ (com $95 \%$ de confiança e $\mathrm{n}$ graus de liberdade) e o erro de efeito. Se o efeito calculado for maior ou igual ao t-crítico, este efeito é considerado significativo e pode ser visualizado em um gráfico de Pareto (Sousa, 2014).

$\mathrm{O}$ valor da distribuição $\mathrm{t}$ de Student é importante para o cálculo da significância dos parâmetros e é definido como a relação entre o valor do parâmetro estimado e o seu desvio padrão (Lima, 2007).

$$
\begin{gathered}
\mathrm{S}^{2}=\left(\mathrm{V}_{1} \mathrm{~S}_{1}{ }^{2}+\mathrm{V}_{2} \mathrm{~S}_{2}{ }^{2}+\ldots+\mathrm{V}_{\mathrm{m}} \mathrm{S}_{2} \mathrm{~m}\right) /\left(\mathrm{V}_{1}+\mathrm{V}_{2}+\ldots+\mathrm{Vm}\right) \\
\mathrm{s}=\sqrt{ } \mathrm{S}^{2} \\
\hat{\mathrm{S}}^{2}=\left(\mathrm{S}^{2} / \mathrm{n}\right) \cdot \sum \mathrm{a}^{2} \\
\hat{\mathrm{S}}=\sqrt{ } \hat{\mathrm{S}}^{2}
\end{gathered}
$$

Onde, vi é o número de graus de liberdade ( $\mathrm{n}-1)$ de cada variância $\left(\mathrm{s}^{2} \mathrm{~m}\right)$ obtido para todos os experimentos para $\mathrm{i}^{\text {th }}$ experimento. $\mathrm{O}$ parâmetro $\mathrm{n}$ é o número de replicatas para cada experimento e a é o coeficiente usado para calcular os efeitos.

A matriz do planejamento experimental $2^{3}$, com três fatores, cada qual a dois níveis é apresentada na tabela 2. 
Tabela 2. Matriz do planejamento experimental $2^{3}$, com três fatores, cada qual a dois níveis

\begin{tabular}{c|r|r|r}
\hline \multirow{2}{*}{ EXPERIMENTO } & \multicolumn{3}{|c}{ VARIÁ VEIS } \\
\cline { 2 - 4 } & Catalisador & Razão Molar & Temperatura \\
\hline $\mathbf{1}$ & +1 & +1 & +1 \\
$\mathbf{2}$ & +1 & +1 & -1 \\
$\mathbf{3}$ & +1 & -1 & +1 \\
$\mathbf{4}$ & +1 & -1 & -1 \\
$\mathbf{5}$ & -1 & +1 & +1 \\
$\mathbf{6}$ & -1 & +1 & -1 \\
$\mathbf{7}$ & -1 & -1 & +1 \\
$\mathbf{8}$ & -1 & -1 & -1 \\
\hline
\end{tabular}

\section{Síntese de biodiesel e cálculo de rendimento}

Com o intuito de selecionar a melhor condição de catalisador, temperatura e razão molar para obtenção de um maior rendimento na síntese de biodiesel, optou-se por realizar um planejamento experimental a dois níveis com três variáveis $\left(2^{3}\right)$, estabelecendo um total de 16 experimentos, contabilizado a réplica, para cada planejamento, conforme descrito no item 2.2 .

A síntese de biodiesel foi realizada em 8 condições experimentais diferentes e em ordem aleatória, realizadas em duplicata, variando a razão molar em 6:1 e 12:1 álcool/óleo, temperatura em $60{ }^{\circ} \mathrm{C}$ e $80{ }^{\circ} \mathrm{Ce}$ catalisadores hidróxido de sódio $(\mathrm{NaOH})$ e hidróxido de potássio $(\mathrm{KOH})$ ambos fixados em $1 \%$ da massa de óleo utilizada. As reações foram processadas no tempo de 1 hora.

Após 1h de reação, o biodiesel foi vertido em funil de separação para separação da fase biodiesel e fase glicerina. Posteriormente, o biodiesel foi conduzido para a etapa de lavagem, com ácido clorídrico 5\%, solução saturada de cloreto de sódio e água destilada.

Após a lavagem, o biodiesel foi filtrado com sulfato de sódio anidro para retirada de umidade (Geris et al. 2007). Depois de concluídas as etapas de purificação, cada reação teve seu rendimento calculado a partir das equações 12 e 13, descritas por Moura (2010).

$$
\mathrm{mbio}_{T}=\frac{m_{\text {óleo } *\left(P M_{\text {óleo }}+3 * P M_{M-P M_{G}}\right.}}{P M_{\text {óleo }}}
$$

Onde:

$M_{\text {óleo }}=$ massa de óleo utilizada na reação,

$\mathrm{PM}_{\text {óleo }}=$ peso molecular do óleo,

$\mathrm{PM}_{\mathrm{M}}=$ peso molecular do metanol

$\mathrm{PM}_{\mathrm{G}}=$ peso molecular do glicerol 
A conversão mássica é calculada pela equação 13.

$$
\text { Conversão }\left(\% \frac{p}{p}\right)=\frac{m^{2} \text { bio }_{\text {exp }}}{m b i o_{T}} * 100
$$

Onde:

Mbio $_{\text {exp }}=$ massa de biodiesel experimental obtido após o processo de purificação.

\section{Resultados e discussão}

O óleo de pequi foi caracterizado quanto a alguns de seus parâmetros físico-químicos mais relevantes para síntese de biodiesel. Os resultados dessa caracterização são apresentados na tabela 3 .

Tabela 3. Propriedades físico-químicas do óleo de pequi utilizado nesse estudo

\begin{tabular}{c|c}
\hline Parâmetros analisados & Óleo de pequi \\
\hline Índice de acidez & $1,28 \mathrm{~g} \mathrm{KOH} / \mathrm{g}$ \\
Porcentagem de ácidos graxos & $0,0 \%$ \\
Índice de saponificação & \\
Densidade & $920 \mathrm{Kg} / \mathrm{m}^{3}$ \\
Umidade e teor de voláteis & $0,039 \%$ \\
\hline
\end{tabular}

De acordo com as análises realizadas os resultados de índice de acidez teve média de 1,28 mg $\mathrm{KOH} / \mathrm{g}$, mostram que a amostra possui acidez menor que o máximo permitido pela legislação para óleo refinado de 2\% Agência Nacional de Vigilância Sanitária (Anvisa, 1999).

Gonçalves et al. (2009) o óleo deve apresentar valores de índice de acidez abaixo de $2 \mathrm{mg}$ de $\mathrm{KOH} / \mathrm{g}$ da amostra, sendo o ideal menor que $1 \mathrm{mg}$ de $\mathrm{KOH} / \mathrm{g}$ da amostra. O resultado do teste de umidade e voláteis mostrou um valor um pouco acima do valor estipulado $(0,2 \%)$ para óleos comestíveis. (Anvisa, 1999).

Segundo Serra, (2010), a porcentagem de ácidos graxos deve ser de no máximo 3\% para se obter um biodiesel de índice de acidez igual ou menor do que $0,5 \mathrm{mg} \mathrm{KOH} / \mathrm{g}$. O presente óleo não apresentou ácidos graxos livres em sua composição.

A densidade de $920 \mathrm{Km} / \mathrm{m}^{3}$ apresentou-se dentro da faixa de valores encontrados para a maioria dos óleos vegetais.

Barbalho et al. (2013), em seu trabalho avaliaram o óleo extraído da polpa de pequi e encontraram valores de 2,89\% de umidade, índice de acidez de 8,49 mg KOH/g e índice de saponificação de 234,71 mg $\mathrm{KOH} / \mathrm{g}$. Os resultados analíticos mostraram que nos óleos, destaca-se a presença dos ácidos graxos oleico e o palmítico, em torno de $50 \%$ para ambos. 
O resultado das análises físico-químicas do óleo de pequi encontrados por (Barbosa et al. 2009) demonstraram que o índice de acidez esteve abaixo de 1\%, índice determinado para o azeite de oliva "Extra virgem" (Anvisa 1999), também apresentaram baixo índice de oxidação mostrado pelo índice de peróxido médio: $1,94 \mathrm{mEq} / \mathrm{kg}$, aproximadamente 5 vezes mais abaixo que o máximo estipulado para óleos comestíveis, $10 \mathrm{mEq} / \mathrm{kg}$ (Anvisa,1999). O resultado do teste de umidade e voláteis mostrou um valor um pouco acima do valor estipulado pela Anvisa (1999) de 0,34\%, este fato pode ter sido causado por fatores relacionado ao processo de extração.

Após a caracterização do óleo e definida a quantidade de experimentos e combinações das variáveis (catalisador, razão molar e temperatura), os biodieseis foram produzidos e o rendimento de cada reação foi calculado. Os resultados obtidos no planejamento fatorial a partir das variáveis estudadas: tipo de catalisador (C), razão molar álcool/óleo (R) e temperatura (T) encontram-se na Tabela 4.

Tabela 4. Resultados de rendimentos obtidos no planejamento fatorial a partir das variáveis estudadas: tipo de catalisador (C), razão molar álcool/óleo (R) e temperatura (T)

\begin{tabular}{r|r|r|r|r}
\hline \multirow{2}{*}{ Experimento } & \multicolumn{3}{|c|}{ Variáveis } & \multirow{2}{*}{ Rendimento } \\
\cline { 2 - 5 } & Catalisador & Razão Molar & Temperatura & \\
\hline $\mathbf{1}$ & $\mathrm{KOH}$ & $12: 1$ & $80^{\circ} \mathrm{C}$ & $41,00 \%$ \\
$\mathbf{2}$ & $\mathrm{KOH}$ & $12: 1$ & $60^{\circ} \mathrm{C}$ & $73,00 \%$ \\
$\mathbf{3}$ & $\mathrm{KOH}$ & $6: 1$ & $80^{\circ} \mathrm{C}$ & $70,00 \%$ \\
$\mathbf{4}$ & $\mathrm{KOH}$ & $6: 1$ & $60^{\circ} \mathrm{C}$ & $69,50 \%$ \\
$\mathbf{5}$ & $\mathrm{NaOH}$ & $12: 1$ & $80^{\circ} \mathrm{C}$ & $37,25 \%$ \\
$\mathbf{6}$ & $\mathrm{NaOH}$ & $12: 1$ & $60^{\circ} \mathrm{C}$ & $63,50 \%$ \\
$\mathbf{7}$ & $\mathrm{NaOH}$ & $6: 1$ & $80^{\circ} \mathrm{C}$ & $50,00 \%$ \\
$\mathbf{8}$ & $\mathrm{NaOH}$ & $6: 1$ & $60^{\circ} \mathrm{C}$ & $69,00 \%$ \\
\hline
\end{tabular}

Observando a tabela 5 nota-se que aparentemente, os maiores rendimentos foram observados quando o catalisador $\mathrm{KOH}$, foi utilizado. Em relação a temperatura e razão molar, os maiores rendimentos foram observados quando essas foram utilizadas em níveis contrários $\left(60^{\circ} \mathrm{C} / 12: 1\right.$ e $\left.80^{\circ} \mathrm{C} / 6: 1\right)$, e ainda assim combinados com o uso do $\mathrm{KOH}$. No entanto só a partir da realização do teste $t(t$ crítico=4,159) e construção do diagrama de Pareto (figura 3), pode-se chegar de fato a uma conclusão sobre as melhores condições experimentais. A partir das respostas obtidas para o rendimento, construiu-se o diagrama de Pareto, como mostra a figura 1. 


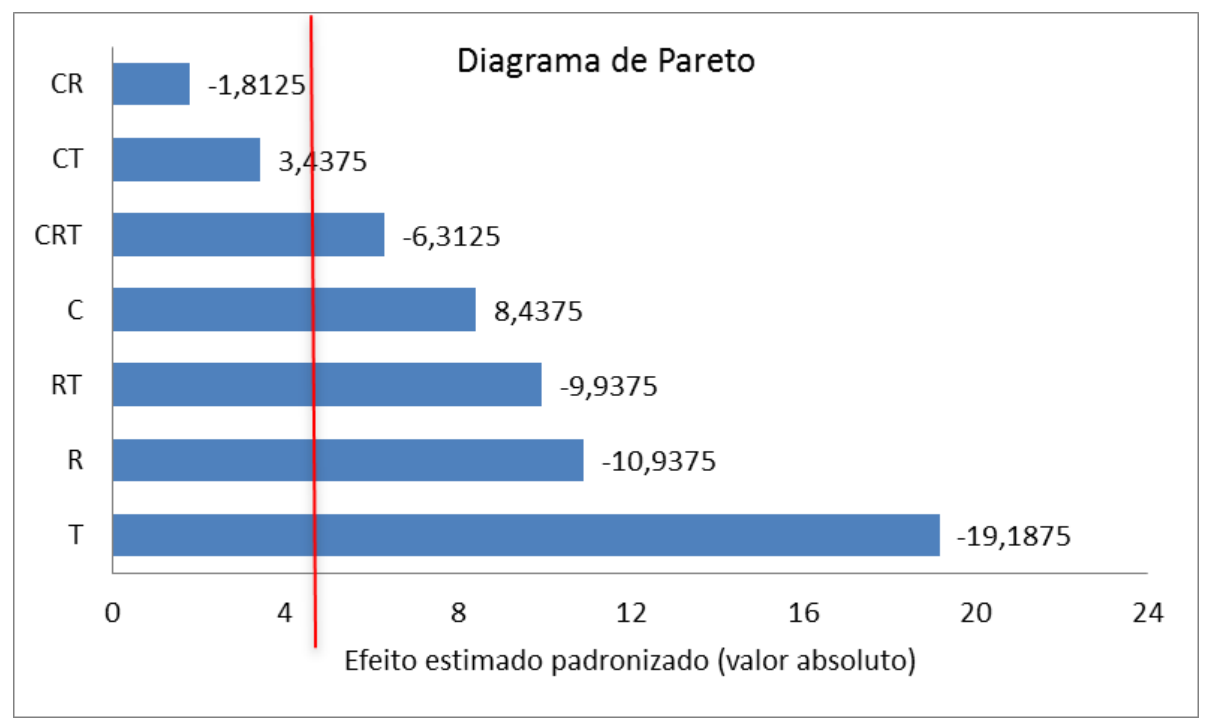

Figura 1: Diagrama de Pareto mostrando a contribuição das variáveis estudadas para o rendimento do biodiesel de óleo de pequi.

O diagrama de Pareto revela os efeitos de primeira, segunda e terceira ordem calculados. Em relação aos efeitos de primeira ordem, observa-se, pelo diagrama de Pareto, que a variável que mais influenciou no aumento do rendimento foi a temperatura. $\mathrm{O}$ sinal negativo referente a $\mathrm{T}$ significa que esta variável quando passa de um nível $+1\left(80^{\circ} \mathrm{C}\right)$ para o nível $-1\left(60^{\circ} \mathrm{C}\right)$, aumenta a resposta (Rendimento da reação). O sinal negativo da variável $\mathrm{R}$ mostra que menores valores na razão molar álcool/óleos promovem maiores rendimentos na reação. Em relação a variável C, o efeito positivo indica que essa variável, em seu maior nível (KOH), aumenta o rendimento da reação.

Em relação aos efeitos de segunda ordem, observa-se que os efeitos das variáveis combinadas CR e CT, apresentaram valores menores (em módulo) que o t crítico calculado, isso significa que os mesmos não provocam efeitos significativos no rendimento da reação (Cunico et al. 2007). Já em relação ao efeito provocado pela combinação das variáveis RT, nota-se que o mesmo é negativo. Observando a equação 6 (apresentada na metodologia), fica claro que para um efeito de segunda ordem apresentar sinal negativo deve-se prevalecer as interações de níveis contrários, ou seja, a combinação entre $R(6: 1)$ e $\mathrm{T}\left(80^{\circ} \mathrm{C}\right)$ ou $\mathrm{R}$ (12:1) e T $\left(60^{\circ} \mathrm{C}\right)$.

Mas finalmente, esse comportamento é de fato comprovado quando se observa o efeito de terceira ordem CRT, onde o mesmo apresenta sinal negativo, o que mostra que na equação 7 apresentada na metodologia, prevaleceu a parcela negativa da equação, sendo possível apenas as interações y---, y++-, y++ ou y-++. No entanto como o único efeito significativo para o catalisador foi positivo $(\mathrm{KOH})$, a única condição que pode ser escolhida é a em que este se encontra em seu nível máximo, ou seja, $\mathrm{KOH}$. Isso indica que as melhores condições de rendimento só podem ser alcançadas nas interações de terceira ordem de y++-, y+-+, em que o catalisador é o $\mathrm{KOH}$ e a temperatura e razão molar devem ser combinadas em níveis contrários, como já suposto anteriormente.

Para chegar a uma conclusão de que condições oferecem uma melhor resposta basta olhar para os efeitos de $\mathrm{T}$ e $\mathrm{R}$ separadamente, para observar que a variável que mais influenciou no aumento do 
rendimento foi a temperatura em seu menor nível $\left(60^{\circ} \mathrm{C}\right)$, como o nível de $\mathrm{T}$ deve ser combinado ao nível contrário de $\mathrm{R}$, a melhor condição experimental capaz de oferecer um maior rendimento na reação de síntese de biodiesel metílico de óleo de pequi a partir da transesterificação alcalina, é aquela em que se combina o catalisador $\mathrm{KOH}$, a temperatura de $60^{\circ} \mathrm{C}$ e uma razão molar metanol/óleo de 12:1.

Apesar dos trabalhos de otimização ser pontuais para cada matéria-prima há uma concordância que a temperatura é o fator mais significativo no processo reacional e que dentro dos intervalos experimentais estudados com diversos óleos vegetais e por diversos autores quanto maior a temperatura menor será o tempo reacional, menor a massa do catalisador e uma maior razão molar álcool-óleo (Lôbo et al. 2007).

Miranda (2015) obteve um aumento de conversão de 5,52\% quando a temperatura reacional foi alterada de 45 para $55{ }^{\circ} \mathrm{C}$, e Liu et al. (2007) em seu experimento com óleo de soja observou um aumento mais ainda significativo da conversão daquele em ésteres metílicos. Um grau de conversão de 33 alterouse para $100 \%$ quando o nível de temperatura passou de 55 para $65{ }^{\circ} \mathrm{C}$. Neste trabalho, ao se comparar dois níveis de temperatura, 60 e $80{ }^{\circ} \mathrm{C}$, verificou-se um efeito contrário ao encontrado por aqueles autores. $\mathrm{O}$ nível de menor valor mostrou-se mais favorável ao melhor rendimento da reação de transesterificação.

No meio reacional há várias substâncias como triglicerídeos, ácidos graxos, metanol e base $(\mathrm{NaOH}$ ou $\mathrm{KOH})$. A reação de transesterificação em meio homogêneo envolve um ataque nucleofílico do grupo metóxi $\left(\mathrm{CH}_{3} \mathrm{O}^{-}\right.$, este formado no meio reacional através da reação do metanol com a hidroxila) ao carbono do grupo da carbonila (eletrófilo) com posterior remoção de uma molécula de glicerol (chamado de grupo retirante) e formação de éster metílico, onde a função da base, $\mathrm{KOH}$ ou $\mathrm{NaOH}$, é prover íons hidroxila $\mathrm{OH}^{-}$ que atuam como catalisadores. Porém, assim como o grupo metóxi, o $\mathrm{OH}^{-}$também pode atuar como nucleófilo através da reação de saponificação e não somente como o desejado que seja exercer a função de catalisador. Quando isto ocorre, há diminuição do grau de conversão de triglicerídeos em ésteres metílicos.

Em uma das etapas do processo de obtenção de biodiesel, dissolve-se certa quantidade de base em metanol, onde este é o solvente. Assim tem-se nesta solução núcleófilos $\mathrm{OH}^{-}$e $\mathrm{MeO}^{-}$(metóxi) solvatados pelas moléculas de metanol. $\mathrm{O}$ íon $\mathrm{OH}^{-}$por ser menor é mais bem estabilizado neste processo de solvatação do que o íon $\mathrm{MeO}^{-}$mais volumoso. Este além de ser uma base mais forte e encontrar-se menos solvatado, ou seja, "mais livre", atua como um nucleófilo mais forte, o que favorece a reação de conversão em ésteres metílicos.

A maior interação entre os íons $\mathrm{OH}^{-}$e as moléculas de metanol no meio reacional reduz a atuação daqueles como nucleófilos. Entretanto, em temperaturas elevadas pode haver quebra destas interações e o $\mathrm{OH}^{-}$tornar-se mais "livre" para realizar ataque nucleofílico através da reação de saponificação, reduzindo assim o rendimento de produção de biodiesel. Isto justifica o resultado analítico do presente trabalho onde as melhores conversões ocorreram no menor nível de temperatura, $60{ }^{\circ} \mathrm{C}$.

A justificativa para o aumento do rendimento com a redução da temperatura no presente trabalho e não nos trabalhos supracitados, se deve ao fato de, em geral com o aumento da temperatura aumenta-se também o grau de conversão de reagentes em produtos devido ao aumento de colisões efetivas entre nucleófilos (grupo metóxi) e eletrófilos (triglicerídeos). Por isso houve aumento de rendimento nos 
experimentos dos outros autores. Contudo em seus trabalhos, os valores de temperatura estudados foram menores, 45 a $55{ }^{\circ} \mathrm{C}$ e 55 a $65{ }^{\circ} \mathrm{C}$, desta maneira, pode-se inferir que nestes níveis não ocorra quebras significativas das interações entre íons $\mathrm{OH}^{-}$e moléculas de metanol para ativação do $\mathrm{OH}^{-}$como agente nucleofílico. $\mathrm{O}$ aumento de temperatura elevou o grau de colisão molecular entre o bom nucleófilo $\left(\mathrm{MeO}^{-}\right.$ ) e o eletrófilo (C-carbonila das moléculas de triglicerídeo) mas não ocasionou o rompimento das interações entre o outro nucleófilo $\left(\mathrm{OH}^{-}\right)$e as moléculas de metanol. No presente trabalho, os níveis estudados foram de 60 e $80{ }^{\circ} \mathrm{C}$, e estes são mais elevados do que os citados anteriormente. Ao se passar do menor para o maior nível, a temperatura alcançada aumenta as colisões moleculares e é capaz de romper a solvatação dos íons $\mathrm{OH}^{-}$dispondo-os para o ataque nucleofílico na reação de saponificação, o que reduz a produção de biodiesel.

Até aqui se explicou os altos rendimentos em menor nível de temperatura neste trabalho considerando apenas o fator de solvatação dos íons $\mathrm{OH}^{-}$. Mas mais um fator explicativo pode ser adicionado: em altas temperaturas o produto formado e o óleo podem ser degradados em moléculas menores e volatilizados, implicando em baixa conversão na reação de transesterificação. Esta degradação depende das características de cada material de partida. Podendo alguns apresentar queda na conversão e outros não, em relação a deterioração térmica. Isto pode também explicar a diferença de resultados obtidos aqui com o óleo de pequi e os resultados obtidos por outros autores que utilizaram um óleo diferente. A mudança de níveis de 45 para $55{ }^{\circ} \mathrm{C}$ e de 55 para $65{ }^{\circ} \mathrm{C}$ não ocasionou aumento de degradação térmica em seus experimentos, mas, neste trabalho, quando se passou de 60 para $80{ }^{\circ} \mathrm{C}$, houve aumento deste fenômeno, que implicou em baixo grau de conversão.

Concluindo, ao se passar de níveis de temperaturas (menor para o maior) em um conjunto não elevado de temperaturas, espera-se que haja aumento de conversão no meio reacional, mas em níveis elevados, devido aos dois fatores anteriormente citados - ativação do nucleófilo $\mathrm{OH}^{-}$e degradação térmica - pode ocorrer decréscimo dos valores de rendimento.

Diante do exposto no presente trabalho, a variável que mais influenciou no aumento do rendimento foi a temperatura em seu menor nível $\left(60^{\circ} \mathrm{C}\right)$, como o nível de $\mathrm{T}$ deve ser combinado ao nível contrário de $\mathrm{R}$, a melhor condição experimental capaz de oferecer um maior rendimento na reação de síntese de biodiesel metílico de óleo de pequi a partir da transesterificação alcalina, é aquela em que se combina o catalisador $\mathrm{KOH}$, a temperatura de $60^{\circ} \mathrm{C}$ e uma razão molar metanol/óleo de 12:1.

\section{Conclusão}

O presente estudo avaliou a influência de três variáveis, catalisador, temperatura e razão molar álcool/óleo, na transesterificação do óleo de pequi, por meio de um planejamento fatorial $2^{3}$, os resultados mostraram que a variável que mais influenciou no aumento do rendimento foi a temperatura em seu menor nível $\left(60^{\circ} \mathrm{C}\right)$.

A partir dos cálculos de efeito da combinação entre as três variáveis, observou-se que os melhores rendimentos são encontrados quando se utiliza o catalisador em seu maior nível $(\mathrm{KOH})$, combinado com 
níveis contrários de razão molar e temperatura. Ou seja, os melhores rendimentos são encontrados quando a transesterificação é realizada utilizando como catalisador o $\mathrm{KOH}$, uma razão molar de 12:1 e uma temperatura de $60^{\circ} \mathrm{C}$, ou como catalisador o $\mathrm{KOH}$, uma razão molar de $6: 1$ e uma temperatura de $80^{\circ} \mathrm{C}$.

\section{Referências bibliográficas}

ANVISA. 1999. Agencia Nacional de Vigilância Sanitária, Resolução RDC No 482, de 23 de setembro de 1999, D.O.U- Diário Oficial da União, Poder Executivo, de 13 de outubro.

BARBALHO, T. C. S; PINHEIRO, A. D. T.; LUCENA, I.L. 2013. Caracterização Físico-Química do Óleo de Pequi (Caryocar brasilienses Camb.). $53^{\circ}$ Congresso Brasileiro de Química. Rio de Janeiro.

BARBOSA, E.A; ANTUNES, R. A. de; FARIAS, T. M; LOPES, N. P. S. 2009. Análise da Qualidade do Óleo de Pequi Produzido e Comercializado no Município de Januária-MG, Brasil. Rev. Bras. De Agroecologia. nov. Vol. 4 No.2

CUNICO, M. W. M; CUNICO, M. M; MIGUEL, O. G; ZAWADZKI, S. F; PERALTA-ZAMORA, P; VOLPATO, N. 2007. Planejamento fatorial: uma ferramenta estatística valiosa para a definição de parâmetros experimentais empregados na pesquisa científica. Visão Acadêmica, Curitiba, v.9, n.1, Jan. Jun.

FERRARI, R.A; OLIVEIRA, V. S; SCABIO, A. 2005. Biodiesel de Soja- Razão de Conversão em Ésteres Etílicos, Caracterização Físico-Química e Consumo e geradores de Energia. Química Nova, c. 28, n. 1.

GERIS, R.; DOS SANTOS, N. A. C; AMARAL, B. A.; MAIA, I. DE S; CASTRO, V. D; CARVALHO, J. R. M. 2007. Biodiesel de soja - reação de Transesterificação para Aulas Práticas de Química Orgânica. Química Nova, v.30 (5), 1369-1373.

GONÇALVES, A.; SOARES, J.; BRASIL, A. N.; NUNES, D. L. 2009. Determinação Do Índice De Acidez De Óleos e Gorduras Residuais Para Produção De Biodiesel. In: Congresso da rede brasileira de tecnologia de biodiesel, 3, 2009, Brasília. Anais... III Congresso da rede brasileira de tecnologia de biodiesel, Brasília, DF, P. 187-188.

KNOTHE, G; GERPEN, J. V; KRAHL, J; RAMOS, L.P. 2006. Manual de biodiesel - São Paulo: Blucher. LIMA, A.; SILVA, A. M. de. O.; TRINDADE, R. A.; TORRES, R. P.; MANCINI-FILHO, J. 2007. Composição química e compostos bioativos presentes na polpa e na amêndoa do pequi (Caryocar brasiliense, Camb.) Rev. Bras. Frutic. 29: 695-698.

LIMA, C. J. B. Produção de Biossurfactante por Pseudomonas aeruginosa empregando óleo de soja residual. Tese de doutorado. Universidade Federal de Uberlândia. Uberlândia.

LIU, X.; HE, H.; WANG, Y.; ZHU, S. Transesterification of soybean oil to biodiesel using SrO as a solid base catalyst. Catalysis Communications, vol. 8, p. 1007- 1111.

LÔBO, I. P.; FERREIRA, S.L.C.; CRUZ, R.S. 2009. Biodiesel: parâmetros de qualidade e métodos analíticos. Quimica nova, v.32 (6), 1596- 1608.

LOPES, P. S. N., PEREIRA, A. V., PEREIRA, E. B. C., MARTINS, E. R., FERNANDES, R. C. 2006. Frutas nativas da região Centro-Oeste do Brasil. Brasília: Embrapa recursos genéticos e biotecnologia.

MIRANDA, S, L. 2015. Produção de Biodiesel a partir da Transesterificação de Óleo de Mamona e Metanol com Catálise Homogênea. Disponível em: $<$ https://uspdigital.usp.br/siicusp/cdOnlineTrabalhoVisualizarResumo?numeroInscricaoTrabalho=4144\& numeroEdicao $=16>$. Acessado em: 01/04.

MORETTO, E; ALVES, R.1986. Óleos e Gorduras Vegetais, Editora da UFSC, Florianópolis.

MOURA, B. S. 2010. Transesterificação Alcalina de Óleos Vegetais Para Produção de Biodiesel: Avaliação Técnica e Econômica. Dissertação apresentada ao curso de Pós-graduação em Engenharia Química da Universidade Federal Rural do Rio de Janeiro, Rio de Janeiro.

SERRA, T.M. 2010. Desenvolvimento De Catalisadores A Base Deestanho (Iv), Para Produção De Ésteres Metílicos De Ácidos Graxos, Via Transesterificação E Esterificação. Dissertação apresentada a Unidade Acadêmica Centro de Tecnologia-UFAL, Maceió.

SOUSA, D. de A. 2014. Ocorrência de poluentes orgânicos persistentes em ovos de Caiman yacare (jacaré-do-Pantanal). Tese apresentada ao Instituto de Química, Universidade Estadual Paulista, Unesp, Araraquara. 